\title{
GMR
}

\section{Cloning, identification, and bioinformatics analysis of a putative aquaporin TsAQP from Trichinella spiralis}

\author{
J.M. Cui ${ }^{1}$, N.Z. Zhang ${ }^{1}$, W.H. Li ${ }^{1}$, H.B. Yan $^{1}$ and B.Q. Fu ${ }^{1,2}$ \\ ${ }^{1}$ State Key Laboratory of Veterinary Etiological Biology, \\ Key Laboratory of Veterinary Public Health of the Ministry of Agriculture, \\ Key Laboratory of Veterinary Parasitology of Gansu Province, \\ Lanzhou Veterinary Research Institute, \\ Chinese Academy of Agricultural Sciences, Lanzhou, China \\ ${ }^{2}$ Jiangsu Co-Innovation Center for Prevention and Control of Important \\ Animal Infectious Disease, Yangzhou, China \\ Corresponding author: B.Q. Fu \\ E-mail: fubaoquan@163.com
}

Genet. Mol. Res. 14 (4): 12699-12709 (2015)

Received March 31, 2015

Accepted June 18, 2015

Published October 19, 2015

DOI http://dx.doi.org/10.4238/2015.October.19.14

\begin{abstract}
Vaccination as a preventative strategy against Trichinella spiralis infection is an ongoing effort, although no ideal vaccine candidates have been identified until now. Identification of more effective antigens that have a role in essential life stages of the parasite and that may be effective vaccine candidates is therefore of importance. In the present study, we identified a novel aquaporin gene (Ts $A Q P)$ from $T$. spiralis, and the potential antigenicity of TsAQP was evaluated by epitope prediction. A total of 11 post-translational modification sites were predicted in the protein and fell into 4 categories: $N$-glycosylation; casein kinase II phosphorylation; protein kinase $\mathrm{C}$ phosphorylation; and $N$-myristoylation sites. TsAQP is a membrane intrinsic protein with high hydrophobicity; the main hydrophobic domains comprised up to $38.5 \%$ of the protein and were distributed at amino acid positions 21-43, 54-71,
\end{abstract}


83-91, 107-121, 163-174, 187-200, and 242-261. The protein consisted mainly of helices $(39.58 \%)$ and loops $(50 \%)$. The advanced structure of TsAQP was predicted using homology modeling, which showed that the protein was formed from 6 membrane-spanning domains connected by 5 loops. Based on these analyses, 6 potential B-cell epitopes and 4 potential T-cell epitopes were further predicted. These results suggest that TsAQP could be a promising antigen candidate for vaccination against $T$. spiralis.

Key words: Trichinella spiralis; Aquaporin; Bioinformatics analysis; Cloning

\section{INTRODUCTION}

Trichinella spiralis is an intracellular parasite and is recognized as one of the crucial pathogens of the zoonotic trichinellosis (Teunis et al., 2012). The parasite has a worldwide distribution and is capable of causing infection in almost all mammals, including humans (Feidas et al., 2014). Human infection is attributed mainly to the consumption of raw or undercooked meat, particularly pork, contaminated with muscle larvae (Dupouy-Camet, 2000). Pathologic lesions in the host are strictly related to the intestinal and muscular parasitic stages, and cause especial harm to the heart and brain (Dupouy-Camet, 2000). In recent years, the re-emergence of trichinellosis in many countries and areas has had an impact on the international commerce of food-producing animals, rekindling public health concerns over the parasite (Djordjevic et al., 2003; Cuperlovic et al., 2005; Blaga et al., 2007).

Albendazole and mebendazole are currently the principal drugs for treating trichinellosis (Gottstein et al., 2009). However, encapsulated larvae in muscle are refractory to treatment with these benzimidazole derivative drugs (Pozio et al., 2003). Investigation of effective vaccination strategies is a potential method to protect against $T$. spiralis infection. Several T. spiralis antigens have been developed and evaluated in mice (Gu et al., 2013; Fang et al., 2014), but none have been found to provide complete protection. Identification of novel functional proteins of $T$. spiralis is therefore greatly desired, for the purposes of developing new specific drugs or vaccines.

The biological membrane system of parasites may interact with receptors in hosts, and is often looked upon as a potential target for the development of drug or vaccine candidates (Kirk, 2004; Landfear, 2010). According to previous studies of Leishmania major and Schistosoma mansoni, Aquaporins, also named water channel proteins (Benga, 2009), are components of this parasite membrane system and are promising candidates for this purpose (Gourbal et al., 2004; Faghiri and Skelly, 2009). Aquaporins (AQPs) are the most abundant proteins in the biological membrane and belong to the major intrinsic protein (MIP) family. The protein group can be divided into water-specific channels (orthodox AQPs), and channels that additionally facilitate transport of glycerol, urea, ammonia, and other small, uncharged molecules (aquaglyceroporins), according to specific functions of each protein. AQPs have been identified in most parasites as having roles in many physiological functions, including osmotaxis; volume regulation; and uptake of nutrients and release of toxic metabolites (Beitz, 2006; Uzcategui et al., 2008; Faghiri et al., 2010). However, little is known about AQPs in $T$. 
spiralis (TsAQP).

In the present study, a putative $T S A Q P$ was amplified from total RNA of $T$. spiralis yunnan isolate. Signal peptides, epitopes, topological structures, and other physical and chemical characteristics of the $T_{S} A Q P$-encoded protein were identified using multiple bioinformatic methods. These findings provide the foundation for our understanding of the biological function of TsAQP, and its potential application in development of epitope-based vaccine or drug.

\section{MATERIAL AND METHODS}

\section{Mice and parasites}

Specific-pathogen-free grade, female Kunming mice ( 8 weeks old) were purchased from the Center of Laboratory Animals at the Lanzhou Institute of Biological Products (Lanzhou, China). Mice were maintained on sterilized food and water and were used for attaining muscle larvae. All procedures involving mice were carried out in accordance with the Animal Ethics Procedures and Guidelines of the People's Republic of China (2010, GB 14925-2010).

Muscle larvae of $T$. spiralis yunnan isolate from infected mice were artifically digested with pepsin- $\mathrm{HCl}$ at 35 days p.i. (Liu et al., 2012), and were purified using the method described by Gamble and Bessonov (Gamble et al., 2000). Muscle larvae were used for total RNA extraction.

\section{Preparation of $T$. spiralis RNA and cloning of $T s A Q P$}

Total RNA was extracted from T. spiralis using Trizol (Invitrogen, USA), according to the manufacturer protocol. First strand cDNA was reverse transcribed using oligo (dT) primers at $42^{\circ} \mathrm{C}$ for $30 \mathrm{~min}$. Then, $T_{s} A Q P$ was amplified using a pair of specific primers AQPPF (5'-GTGAGCAGAGCATATTCACA-3') and AQPPR (5'-GGGGCTTTAGAAATGTGAGA $-3^{\prime}$ ) by PCR. Individual reactions for PCR amplification contained $1.0 \mu \mathrm{L}$ cDNA; $2.0 \mu \mathrm{L} 2.5 \mathrm{mM}$ dNTP mix; $2.5 \mu \mathrm{L}$ 10X PCR buffer; $1.0 \mu \mathrm{L} 10 \mu \mathrm{M}$ of each primer; $0.4 \mu \mathrm{L} 2.5 \mathrm{U} / \mathrm{mL}$ Taq DNA polymerase; and $17.1 \mu \mathrm{L} \mathrm{ddH_{2 }}$ O. Cycling conditions for PCR were: 5 min at $94^{\circ} \mathrm{C} ; 35$ cycles of $94^{\circ} \mathrm{C}$ for $1 \mathrm{~min}, 56^{\circ} \mathrm{C}$ for $1 \mathrm{~min}$, and $72^{\circ} \mathrm{C}$ for $1 \mathrm{~min} 30 \mathrm{~s}$; and a final extension at $72^{\circ} \mathrm{C}$ for $10 \mathrm{~min}$.

Products of PCR $(5 \mu \mathrm{L})$ were analyzed by electrophoresis on $1.0 \%$ agarose $(w / v)$ gel stained with ethidium bromide. The gel was visualized using a gel ImageScanner system (PeiQing, Science \& Technology, Shanghai, China).

\section{Cloning, sequencing, and identification of $T s A Q P$}

The target gene fragment was purified using a gel extraction kit (TIANGEN, China) according to the manufacturer protocol. The fragment was ligated into a pMD18-T vector (TaKaRa, China), and transformed into Escherichia coli DH5a competent cells, which were then coated onto plates of Luria-Bertani (LB) supplemented with ampicillin (AMP, $100 \mu \mathrm{g} /$ $\mathrm{mL}$ ), and cultured at $37^{\circ} \mathrm{C}$. Twelve hours later, 10 single colonies were selected for culture in LB liquid medium containing $100 \mu \mathrm{g} / \mathrm{mL}$ AMP, and shaken overnight at $37^{\circ} \mathrm{C}$. Colonies were then screened by PCR amplification, and positive colonies were sent for sequencing to GENEWIZ $^{\mathrm{TM}}$ (China). Sequencing results were aligned on NCBI (http://www.ncbi.nlm.nih. gov/BLAST/). Positive recombinant plasmid was termed pMD-TsAQP. 


\section{Analysis of TsAQP amino acid sequence}

General features of TsAQP including molecular weight, isoelectric point, amino acid (aa) composition, molecular extinction coefficient, and half-life were predicted using the ProtParam software (http://web.expasy.org/protparam/). Motifs were identified using the MotifScan software (http://myhits.isb-sib.ch/cgi-bin/motif_scan), and hydrophilicity/ hydrophobicity was analyzed using ProtScale.

The secondary structure of TsAQP was predicted using PredictProtein (https://www. predictprotein.org/). Then, determination of the topologic structural characteristics of TsAQP was performed using the online service SMART (Letunic et al., 2012), and the 3-dimensional (3D) structure estimated by homology modeling using the online service SWISS-MODEL (Kiefer et al., 2009). TsAQP was constructed via the automated modeling program using the software Swiss PDB-Viewer and the corresponding protein from Plasmodium falciparum as a template (code: 3c02). A 3D model of TsAQP was constructed using the program Swiss-PDB Viewer (Guex et al., 2009), and assessed using Verify_3D (http://services.mbi.ucla.edu/Verify_3D/).

The flexibility and accessibility of the structural proteins were analyzed by KarplusSchulz and Plot-Emini methods, respectively, using the DNAStar 7.1 software (DNAStar, USA). Finally, the potential linear B-cell epitopes of TsAQP were predicted with the JamesonWolf index using the DNAStar software. The potential T cell epitopes were calculated using the online server SYFPEITHI (http:www.syfpE-thi.de/Scripts/MHCServer.dll/EpitopePrediction. htm) and Propred I (http:www.imtech.res.in/raghava/propred1/).

\section{RESULTS}

\section{Identification of recombinant vector pMD-TsAQP}

The recombinant plasmid pMD-TsAQP was identified by PCR. Agarose gel electrophoresis revealed the amplified fragment was approximately $1100 \mathrm{bp}$; this was consistent with the expected length (Figure 1). The aa sequence translated from the $T_{S} A Q P$ open reading frame (ORF) demonstrated $45 \%$ identity to human AQP9. This indicated that insertion into the pMD18-T vector was successful.

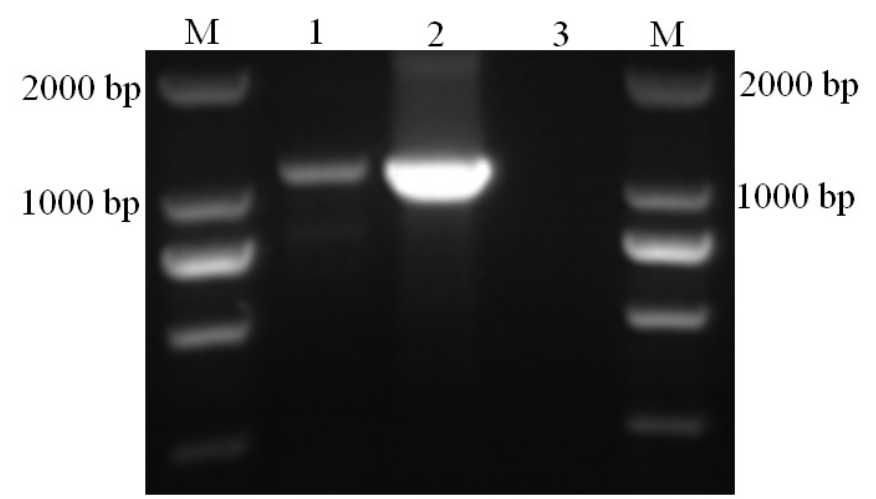

Figure 1. Identification of the recombinant vector pMD-TsAQP by polymerase chain reaction amplification. Lane $M=$ DL2000 DNA markers; lane 1 = PCR products of cDNA TsAQP; lane 2 = identification of pMDTsAQP by PCR; lane 3 = control. 


\section{Physiological and biochemical characters of TsAQP}

The ORF of $T_{s} A Q P$ was found to be $867 \mathrm{bp}$ in length, and encoded a 288 -aa protein (Figure 2). The molecular weight of the TsAQP protein was $31 \mathrm{kDa}$, and its theoretical isoelectric point was 8.47 . At $280 \mathrm{~nm}$ in water, the molar extinction coefficient of TsAQP was $52,035 \mathrm{~mol} / \mathrm{cm}$. The half-life of the protein in yeast and Escherichia coli was estimated as $>20$ and $>10 \mathrm{~h}$, respectively. TsAQP protein had an instability index of 32.48 , and could therefore be classified as stable.

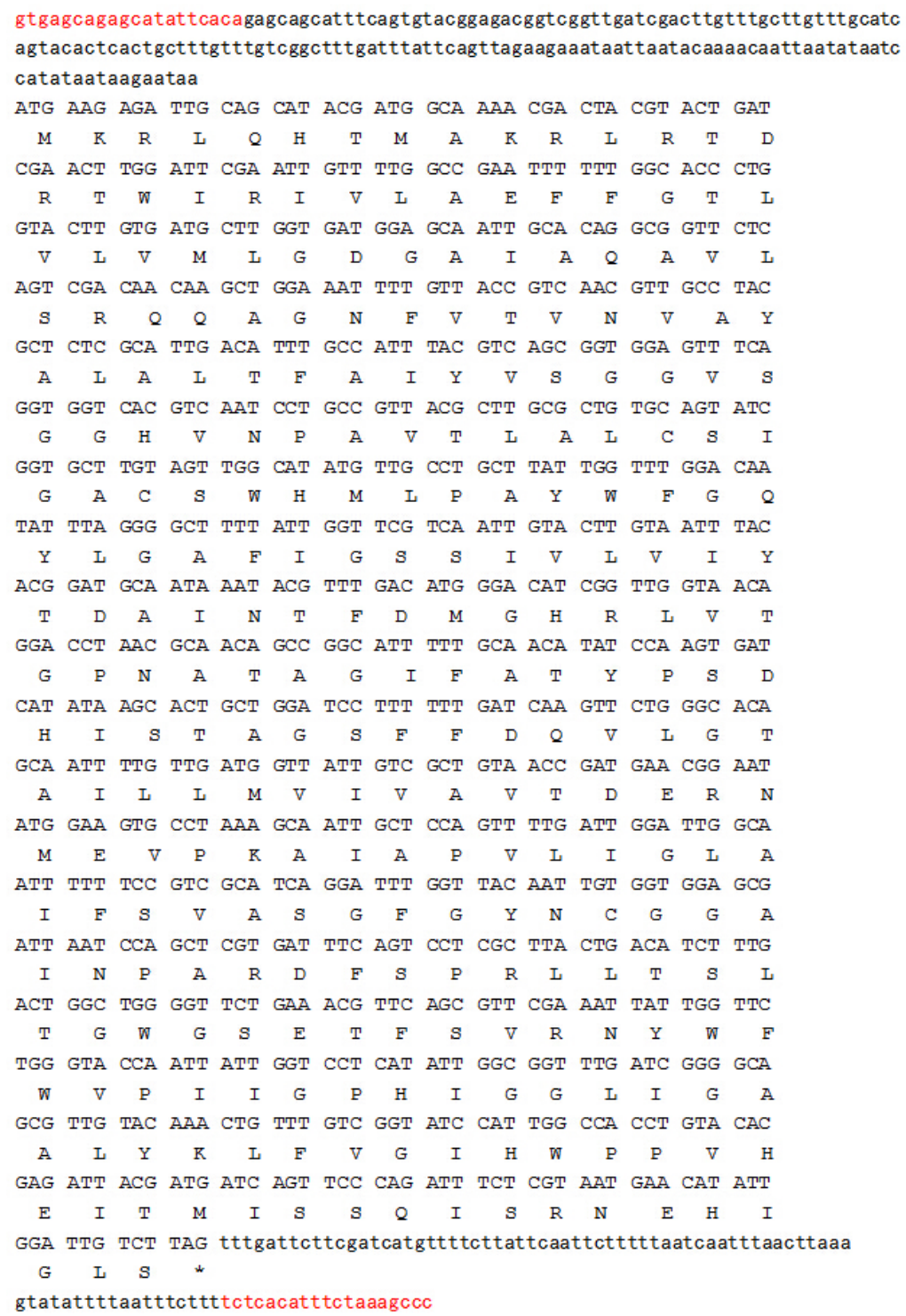

Figure 2. Nucleotide and amino acid sequences of TsAQP. 


\section{Prediction of functional motifs}

After alignment of TsAQP with other identified AQPs, the MIP family signature was predicted at position 78-86, and a cysteine residue at position 207. Some other post-translational modification sites, such as one $\mathrm{N}$-glycosylation, two casein kinase II phosphorylation, three protein kinase $\mathrm{C}$ phosphorylation, and five $N$-myristoylation sites, were also predicted in TsAQP (Table 1).

Table 1. Post-translational modification (PTM) sites of the putative TsAQP protein.

\begin{tabular}{llc}
\hline PTM sites & Position & Numbers \\
\hline$N$-glycosylation & $138-141$ & 1 \\
Casein kinase II phosphorylation & $157-160,280-283$ & 2 \\
$N$-myristoylation & $51-56,72-77,104-109,142-147,250-255$ & 5 \\
Protein kinase C phosphorylation & $14-16,218-220,234-236$ & 3 \\
\hline
\end{tabular}

\section{Prediction of hydrophilicity/hydrophobicity and secondary structure of TsAQP}

Hydrophobic effect is a dominant force for protein folding and architectural stability (Malleshappa Gowder et al., 2014). The TsAQP protein was predicted to be characteristically hydrophobic, with an aliphatic index of 107.36, and grand average of hydropathicity of 0.558. Further analysis of the hydrophilic/hydrophobic regions of TsAQP, by the Kyte \& Doolittle selection of Hphob within the ProtScale software program, indicated that the protein contained multiple hydrophobic regions (38.5\%). High-scoring hydrophobic domains were predicted at aa positions 21-43, 54-71, 83-91, 107-121, 163-174, 187-200, and 242-261 (Figure 3).

PredictProtein results calculated the proportion of $\alpha$-helixes, strands, and random coils in TsAQP as $39.58,10.42$, and 50\%, respectively.

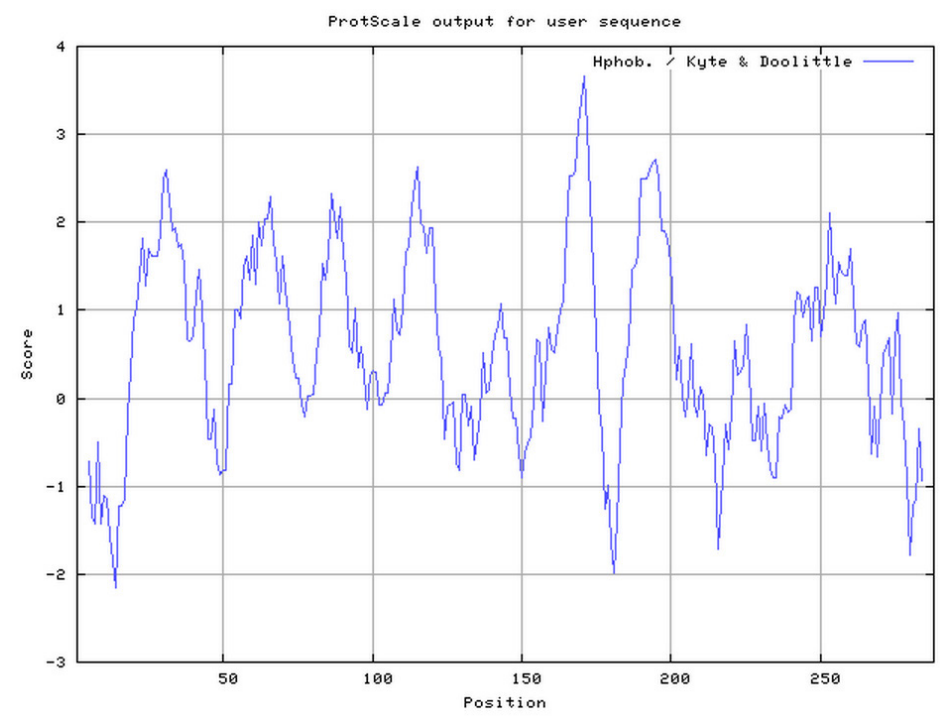

Figure 3. Prediction of hydrophobic domains of TsAQP. 


\section{Homology modeling of TsAQP}

The tertiary structure of a protein always reveals several specific biological functions. Prediction of 3D protein structure, based on homologous models using a protein structure modeling strategy, is currently a popular method and has the benefits of being easier, faster, and more efficient than traditional physical and biochemical methods, such as X-ray crystallography and nuclear magnetic resonance (Biasini et al., 2014).

In the current study, TsAQP was found to consist of 6 membrane-spanning domains connected by 5 loops (Figure 4), which corresponded to the typical structure of aquaporin (AQP1, PfAQP). Analysis of the quality of the TsAQP model using Verify 3D online software showed that $87.92 \%$ of the residues had an averaged $3 \mathrm{D} / 1 \mathrm{D}$ score $\geq 0.2$, indicating the favored $3 \mathrm{D}$ structure of the protein was constructed.

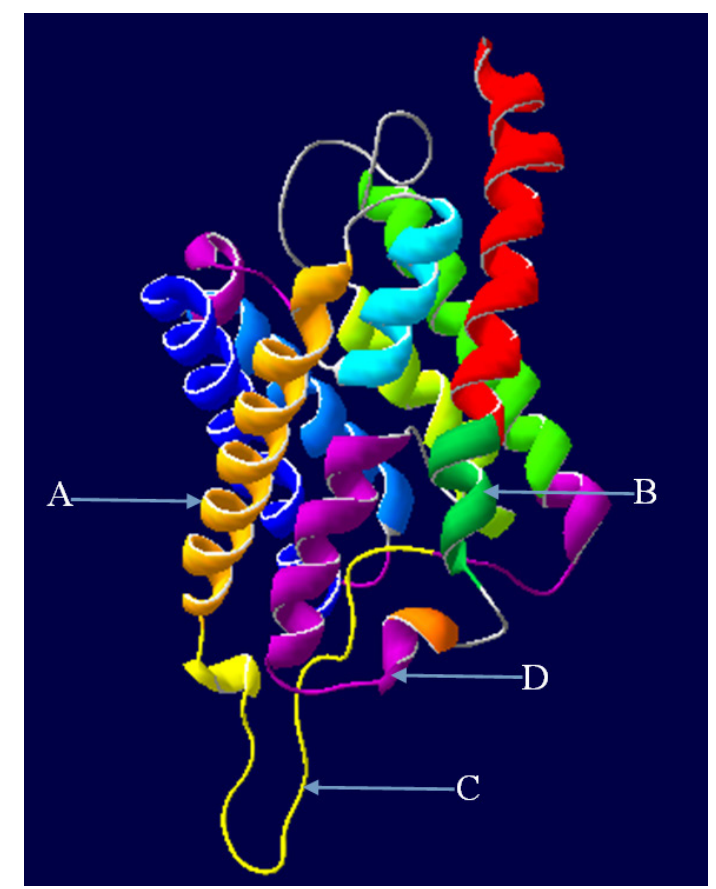

Figure 4. Tertiary structure prediction of TsAQP protein. Ribbon diagram of the $\alpha$-helix (A), $\beta$-fold (B), random coil (C), partial potential epitopes (D; purple).

\section{Potential B or T cell epitopes}

$\mathrm{B}$ or $\mathrm{T}$ cell epitopes are usually discerned by the host immune system, and have the potential to induce protective immunity. Integrating consideration of the characters of variability, fragment mobility, hydrophilicity, surface accessibility and secondary structures, a total of 6 potential B cell epitopes of TsAQP were predicted (Table 2).

Based on integration of results from SYFPEITHI and Propred I software, 4 dominant $\mathrm{T}$ cell epitopes for alleles HLA-A*0201 were predicted; these were located at aa positions $22-$ 30, 110-118, 167-175, and 191-199 (Figure 5). 
Table 2. Potential B cell epitopes position and sequences.

\begin{tabular}{lcl}
\hline Numbers & Position & Potential B cell epitopes sequences \\
\hline 1 & $11-16$ & RLRTDR \\
2 & $46-51$ & SRQQAG \\
3 & $71-78$ & SGGVSGGH \\
4 & $147-157$ & YPSDHISTAGS \\
5 & $213-232$ & PARDFSPRLLTSLTGWGSET \\
6 & $276-283$ & SSQISRNE \\
\hline
\end{tabular}

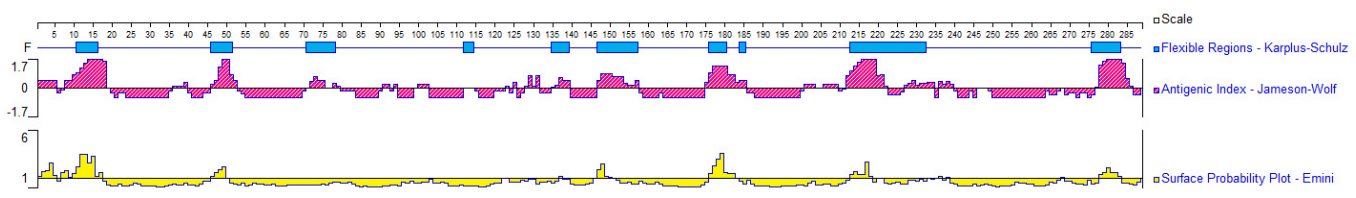

Figure 5. Analysis of TsAQP. Antigenic index (red), flexible regions (F; blue), and surface probability (yellow).

\section{DISCUSSION}

AQPs form homotetramers embedded in the lipid bilayer, and each monomer has an independent functional pore facilitating passive transport of water and/or other small neutral molecules (Gomes et al., 2009). These proteins are found in virtually all organisms, from vertebrates to invertebrates and even viruses, and are involved in various life processes (Hachez and Chaumont, 2010). However, due to a lack of genomic sequencing data, it was not previously known whether the conservative protein exists in T. spiralis. In the present study, the potential AQP was cloned in T. spiralis, and its physiological and biochemical characters and epitopes were predicted.

Four short single-read transcript sequences of the AQP gene were detected in $T$. spiralis EST database, but TSAQP was not annotated in the genomic sequencing data. We speculated that TsAQP lacked a classical signal sequence and was chimeric with putative kinesin motor protein, which lead to an erring annotation. Here, the cloned cDNA fragment of $T s A Q P$ was $1134 \mathrm{bp}$, and encoded a 288 aa protein with $45 \%$ identity to the AQP9 protein in humans. This suggests that TsAQP is an aquaglyceroporin, and may function to transport other molecules in addition to water.

There are two constriction points in AQPs. The narrowest pore mouth is termed the aromatic arginine $(\operatorname{ar} / \mathrm{R})$ constriction and is nearly the size of a single water or glycerol molecule (Campbell et al., 2008). In Fasciola gigantica AQPs, the Cys204 at the pore mouth is replaced by Tyr, which leads to a loss of water permeability (Geadkaew et al., 2011). The second constriction is located in the center of the channel formed by the NPA motifs, and has the ability to transport water molecules, but prevent leaking of proteins (Campbell et al., 2008). In P. falciparum, the AQP protein is comprised of an NLA/NPS motif, and mutation of this motif to NPS/NLA resulted in complete ablation of protein function (Hedfalk et al., 2008). Phosphorylation, $\mathrm{pH}$ and $\mathrm{Ca}^{2+}$ levels can also directly influence the permeability of many AQP proteins (Campbell et al., 2008).

Bioinformatics is becoming increasingly significant in the prediction of protein structure, function, and some other biological characteristics, and has the benefits of being effective and 
low-cost compared to traditional methods (Yang and Yu, 2009; Zhang et al., 2014). In the current study, prediction of functional domains revealed that TsAQP contained a MIP family signature at position 78-86 and a cysteine residue at position 207. Studies of AQPs from humans and S. mansoni have demonstrated that MIP and cysteine residue are responsible for binding to inhibitors (for example, mercuric chloride), which may be potential drug targets (Kuwahara et al., 1997; Faghiri and Skelly, 2009). Phosphorylation of AQPs by protein kinase (PK), which can greatly affect their transport properties and subcellular localization, has been detected in both plants and animals (Hachez and Chaumont, 2010). Here, 3 PK C phosphorylation sites were identified in TsAQP at the position of 14-16, 218-220, and 234-236.

In analysis of protein secondary structures, $\alpha$-helixes and $\beta$-sheets are considered to play roles in cell stability, mechanical signaling, and tissue constitution, whereas random coils are exposed to the protein surface (Perticaroli et al., 2014). In the present study, TsAQP protein was shown to consist mainly of $\alpha$-helixes and random coils (approximately $90 \%$ ), and to have good stability. To visualize secondary structures for analysis, the $3 \mathrm{D}$ model of TsAQP was also constructed by homologous modeling. This showed that the protein was consisted by 6 main transmembrane helices. The two NPA motifs located in the central channel as pseu-half transmembrane domains. The putative ar/R constriction at the narrowest pore mouth, and NPA motifs, were also predicted in TsAQP.

Linear $\mathrm{T}$ and $\mathrm{B}$ cell epitopes play important roles in inducing an acquired immune response, which holds undoubted potential for vaccine design (Yang and Yu, 2009; Flower, 2013). The ability to predict immunogenicity of unknown proteins, and identify antigen epitopes suitable for vaccine development, using bioinformatic techniques is both extremely useful and economical (Yang and Yu, 2009; Song and He, 2012). Combined with hydrophilicity/ hydrophobicity, antigen index and surface accessibility analyses, 6 potential B cell epitopes, and 4 potential $\mathrm{T}$ cell epitopes were predicted in TsAQP.

In conclusion, a putative $T S A Q P$ was cloned in T. spiralis, and the encoded protein was predicted to be a multi-functional water channel protein, indicating an important role for the protein in the life cycle of this parasite. Further investigations of the potential for use of this protein as an antigenic target in vaccine and drug development, will extend our understanding of trichinellosis control. To this end, studies in suitable experimental animal models of $T$. spiralis infection are warranted.

\section{Conflicts of interest}

The authors declare no conflict of interests.

\section{ACKNOWLEDGMENTS}

Research supported was provided by the National Natural Science Foundation of China (grant \#31272555).

\section{REFERENCES}

Animal Ethics Procedures and Guidelines (2010). Laboratory Animal. Requirements of environment and housing facilities. People's Republic of China. GB 14925-2010.

Beitz E (2006). Aquaporin water and solute channels from Malaria parasites and other pathogenic protozoa. Chem. Med. Chem. 1: 587-592. 
Benga G (2009). Water channel protein (later called aquaporins) and relatives: past, present, and future. IUBMB Life 61: 112-133.

Biasini M, Bienert S, Waterhouse A, Arnold K, et al. (2014). SWISS-MODEL: modelling protein tertiary and quaternary structure using evolutionary information. Nucleic Acids Res. 42: W252-W258.

Blaga R, Durand B, Antoniu S, Gherman C, et al. (2007). A dramatic increase in the incidence of human trichinellosis in Romania over the past 25 years: impact of political changes and regional food habits. Am. J. Trop. Med. Hyg. 76: 983-986.

Campbell EM, Ball A, Hoppler S and Bowman AS (2008). Invertebrate aquaporins: a review. J. Comp. Physiol. B 178: 935-955.

Cuperlovic K, Djordjevic M and Pavlovic S (2005). Re-emergence of trichinellosis in southeastern Europe due to political and economic changes. Vet. Parasitol. 132: 159-166.

Djordjevic M, Bacic M, Petricevic M, Cuperlovic K, et al. (2003). Social, political, and economic factors responsible for the reemergence of trichinellosis in Serbia: a case study. J. Parasitol. 89: 226-231.

Dupouy-Camet J (2000). Trichinellosis: a worldwide zoonosis. Vet. Parasitol. 93:191-200.

Faghiri $\mathrm{Z}$ and Skelly PJ (2009). The role of tegumental aquaporin from the human parasitic worm, Schistosoma mansoni, in osmoregulation and drug uptake. FASEB J. 23: 2780-2789.

Faghiri Z, Camargo SM, Huggel K, Forster IC, et al. (2010). The tegument of the human parasitic worm Schistosoma mansoni as an excretory organ: the surface aquaporin SmAQP is a lactate transporter. PloS One 5: e10451.

Fang L, Sun L, Yang J, Gu Y, et al. (2014). Heat shock protein 70 from Trichinella spiralis induces protective immunity in BALB/c mice by activating dendritic cells. Vaccine 32: 4412-4419.

Feidas H, Kouam MK, Kantzoura V and Theodoropoulos G (2014). Global geographic distribution of Trichinella species and genotypes. Infect. Genet. Evol. 26: 255-266.

Flower DR (2013). Designing immunogenic peptides. Nat. Chem. Biol. 9: 749-753.

Gamble HR, Bessonov AS, Cuperlovic K, Gajadhar AA, et al. (2000). International Commission on Trichinellosis: recommendations on methods for the control of Trichinella in domestic and wild animals intended for human consumption. Vet. Parasitol. 93: 393-408.

Geadkaew A, Von Bülow J, Beitz E, Grams SV, et al. (2011). Functional analysis of novel aquaporins from Fasciola gigantica. Mol. Biochem. Parasit. 175: 144-153.

Gomes D, Agasse A, Thiébaud P, Delrot S, et al. (2009). Aquaporins are multifunctional water and solute transporters highly divergent in living organisms. Biochim. Biophys. Acta 1788: 1213-1228.

Gottstein G, Pozio E and Nöckler K (2009). Epidemiology, diagnosis, treatment, and control of trichinellosis. Clin. Microbiol. Rev. 22: 127-145.

Gourbal B, Sonuc N, Bhattacharjee H, Legare D, et al. (2004). Drug uptake and modulation of drug resistance in Leishmania by an aquaglyceroporin. J. Biol. Chem. 279: 31010-31017.

Gu Y, Wei JF, Yang J, Huang JJ, et al. (2013). Protective immunity against Trichinella spiralis infection induced by a multi-epitope vaccine in a murine model. PLoS One 8: e77238.

Guex N, Peitsch MC and Schwede T (2009). Automated comparative protein structure modeling with SWISS-MODEL and Swiss-PdbViewer: a historical perspective. Electrophoresis 30: S162-S173.

Hachez C and Chaumont F (2010). Aquaporins: A family of highly regulated multifunctional channels. Adv. Exp. Med. Biol. 679: 1-17.

Hedfalk K, Pettersson N, Oberg F, Hohmann S, et al. (2008). Production, characterization and crystallization of the Plasmodium falciparum aquaporin. Protein Expr. Purif. 59: 69-78.

Kiefer F, Arnold K, Künzli M, Bordoli L, et al. (2009). The SWISS-MODEL Repository and associated resources. Nucleic Acids Res. 37: D387-D392.

Kirk K (2004). Channels and transporters as drug targets in the Plasmodium-infected erythrocyte. Acta Trop. 89: 285-294.

Kuwahara K, Gu Y, Ishibashi K, Marumo F, et al. (1997). Mercury-sensitive residues and pore site in AQP3 water channel. Biochemistry 36: 13973-13978.

Landfear SM (2010). Transporters for drug delivery and as drug targets in parasitic protozoa. Clin. Pharmacol. Ther. 87: $122-125$.

Letunic I, Doerks T and Bork P (2012). SMART 7: recent updates to the protein domain annotation resource. Nucleic Acids Res. 40: D302-D305.

Liu X, Song Y, Jiang N, Wang J, et al. (2012). Global gene expression analysis of the zoonotic parasite Trichinella spiralis revealed novel genes in host parasite interaction. PLoS Negl. Trop. Dis. 6: e1794

Malleshappa Gowder S, Chatterjee J, Chaudhuri T and Paul K (2014). Prediction and analysis of surface hydrophobic residues in tertiary structure of proteins. Sci. World J. 2014: 071258. 
Perticaroli S, Nickels JD, Ehlers G and Sokolov AP (2014). Rigidity, secondary structure, and the universality of the Boson Peak in proteins. Biophys. J. 106: 2667-2674.

Pozio E, Sacchini D, Sacchi L, Tamburrini A, et al. (2003). Failure of mebendazole in the treatment of humans with Trichinella spiralis infection at the stage of encapsulating larvae. Clin. Infect. Dis. 32: 638-642.

Song J and He QF (2012). Bioinformatics analysis of the structure and linear B-cell epitopes of aquaporin-3 from Schistosoma japonicum. Asian Pac. J. Trop. Med. 5: 107-109.

Teunis PF, Koningstein M, Takumi K and van der Giessen JW (2012). Human beings are highly susceptible to low doses of Trichinella spp. Epidemiol. Infect. 140: 210-218.

Uzcategui NL, Zhou Y, Figarella K, Ye J, et al. (2008). Alteration in glycerol and metalloid permeability by a single mutation in the extracellular C-loop of Leishmania major aquaglyceroporin LmAQP1. Mol. Microbiol. 70: 1477-1486.

Yang X and Yu X (2009). An introduction to epitope prediction methods and software. Rev. Med. Virol. 19: 77-96.

Zhang NZ, Huang SY, Zhou DH, Xu Y, et al. (2014). Identification and bioinformatic analysis of a putative calciumdependent protein kinase (CDPK6) from Toxoplasma gondii. Genet. Mol. Res. 13: 10669-10677. 